\title{
Finite temperature simulation studies of spin-flop magnetic random access memory devices
}

\author{
S.T. Chui ${ }^{\mathrm{a}, *}$, Ching-Ray Chang ${ }^{\mathrm{b}}$ \\ ${ }^{a}$ Bartol Research Institute, University of Delaware, Newark, DE 19716, USA \\ ${ }^{\mathrm{b}}$ Department of Physics, National Taiwan University, Taipei, Taiwan
}

Received 31 October 2005; received in revised form 23 December 2005; accepted 24 December 2005

Available online 4 January 2006

Communicated by R. Wu

\begin{abstract}
Spin-flop structures are currently being developed for magnetic random access memory devices. We report simulation studies of this system. We found the switching involves an intermediate edge-pinned domain state, similar to that observed in the single layer case. This switching scenario is quite different from that based on the coherent rotation picture. A significant temperature dependence of the switching field is observed. Our result suggests that the interplane coupling and thus the switching field has to be above a finite threshold for the spin-flop switching to be better than conventional switching methods.
\end{abstract}

(C) 2005 Elsevier B.V. All rights reserved.

There has been much interest recently in the micromagnetics of non-volatile Magnetic Random Access Memory (MRAM) devices. Both individual and small arrays of MRAM devices have been demonstrated to have a fast access speed $(\approx 2 \mathrm{~ns})$, low power consumption, density scalability, and compatibility with semiconductor processing. In a functional high-density MRAM chip, $100 \%$ bit selectivity is required. Less than $100 \%$ selectivity means a poor yield and therefore a high production cost. High bit selectivity means a high degree of control of magnetic switching in millions of MRAM devices.

To tackle the bit selectivity problem, a new approach to the design of MRAM's has recently been proposed and implemented in 4 Mbit MRAM by the Motorola group [1]. They considered an antiferromagnetically coupled bilayer film. The information is stored in the magnetization of one of the layers. The magnetization of this layer is changed by magnetic fields oriented at different angles at different times, motivated by the coherent rotation picture. In spite of the great success in selectivity in current demonstration of these MRAM devices,

\footnotetext{
* Corresponding author.

E-mail address: chui@udel.edu (S.T. Chui).
}

the fields required for the switching is quite high and, based on the coherent rotation picture, is expected to get higher for smaller samples. This approach will be even more useful if the switching field can be reduced. To address these questions, it is important to understand more carefully the switching mechanism and the validity of the coherent rotation picture for these type of structures [2-4]. To explore these issues we have performed Monte Carlo simulation of the switching at different temperatures and find a significant temperature dependence of the switching field, different from the expectation of the coherent rotation model. Examination of the magnetic configurations suggest that the switching mechanism is different from that of the simple coherent rotation picture. The switching involves going through an intermediate state with edge-pinned domains and is very similar to that observed in the single layer case [5]. In addition, we found that the interplane coupling and thus the switching field has to be above a finite threshold for the spinflop switching to be better than the conventional switching. We next describe our results in detail.

We first recapitulate the switching process proposed. At zero field, the top (bottom) magnetization $M_{1}\left(M_{2}\right)$ are antiparallel. Under an external field $M_{1}$ and $M_{2}$ are no longer completely antiparallel and they mostly are aligned perpendicular to $H$. 

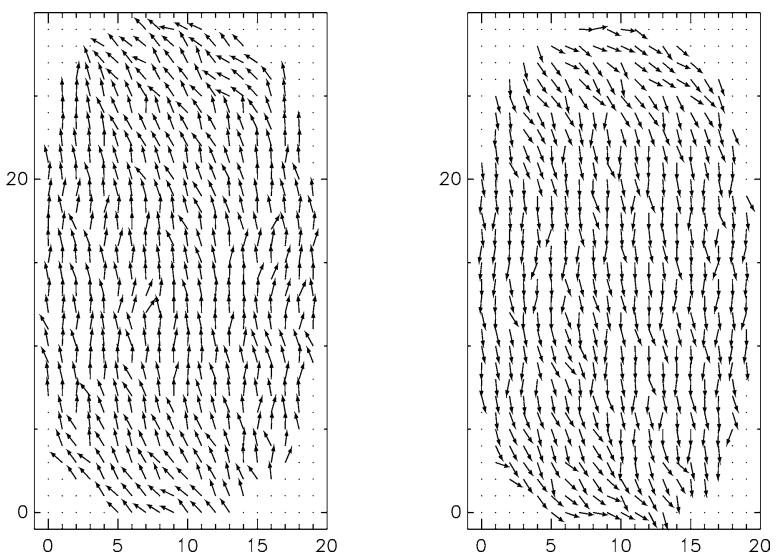

Fig. 1. A snap shot of the projection of the magnetization configuration onto the $x y$ plane at zero field. The left (right) panel is for the top (bottom) layer. Only every other spins in the $x$ and the $y$ directions are shown.

At increasing times the external field is rotated. The magnetizations follow its direction and as a result are rotated. Our simulation results suggest a picture very different from this.

The present calculation follows our earlier studies on bilayer systems [6] where we focused on the case of a rectangular element with the external field along the easy axis of the element. The interaction energy between the spins at the positions $\mathbf{R}, \mathbf{R}^{\prime}$ is $U=0.5 \sum_{i, j=x, y, z} \sum_{\mathbf{R R}^{\prime}} V_{i j}\left(\mathbf{R}-\mathbf{R}^{\prime}\right) S_{i}(\mathbf{R}) S_{j}\left(\mathbf{R}^{\prime}\right)-$ $\sum_{i} S_{i} \cdot H-2 K \sum_{i} S_{i x}^{2}$ where $V=V^{d}+V^{e}$ is the sum of the dipolar energy $V_{i j}^{d}(\mathbf{R})=g_{a b} \nabla_{i} \nabla_{j}\left(1 /\left|\mathbf{R}_{a b}\right|\right)$ and the nearest neighbor exchange energy $V^{e}=-J \delta\left(\mathbf{R}=\mathbf{R}^{\prime}+a\right) \delta_{i j}$. Here $a$ denotes the nearest neighbour distance. The exchange energy includes an intralayer ferromagnetic exchange of magnitude $J$ and an interlayer antiferromagnetic exchange of magnitude $J_{i}$. $g_{a b}=M_{a} M_{b} / v$ where $M_{a}$ is the magnetization of site $a, v$ is the unit cell volume.

Our Monte Carlo calculation produces known results in micromagnetics in all situations that we have tested [7]. Thus we feel confident that the Monte Carlo technique produces reliable results for the coercive behaviour of magnetic systems. Our calculation is carried out on classical three-dimensional spins (the "Heisenberg" model) under free boundary conditions. Typical runs take 16000 Monte Carlo steps/spin.

The zero field spin configuration for an elliptically shaped permalloy (with exchange $A=1.3 \times 10^{-6} \mathrm{erg} / \mathrm{cm}$, magnetization $M_{s}=800 \mathrm{emu} / \mathrm{cc}$ and anisotropy constant $K=5 \times$ $10^{3} \mathrm{erg} / \mathrm{cc}$ ) sample is illustrated in Fig. 1. To illustrate typical behaviour we have carried out calculations for a sample of dimension $0.4 \mu \mathrm{m} \times 0.6 \mu \mathrm{m} \times 50 \AA$; the ratio of the interlayer to the intralayer exchange is $J_{i} / J=0.031$; the spacing between the layers, $60 \AA$.

The magnetization is not uniform. There are domains near the top and bottom of the $y$ axis. If these domains did not form, there will be magnetic charges $q_{M}=-\nabla \cdot M$ formed at the boundary of the element. $q_{M}$ is largest near the vertical ( $\left.y\right)$ axis and zero at the $x$ axis. We thus expect the edge domains to form near the $y$ axis to reduce the magnetostatic energy. In our simulation, we first apply a field of magnitude $H$ along the direction $\mathbf{u}=(-1,-1) / \sqrt{2}$. After equilibrium is reached, the final con-
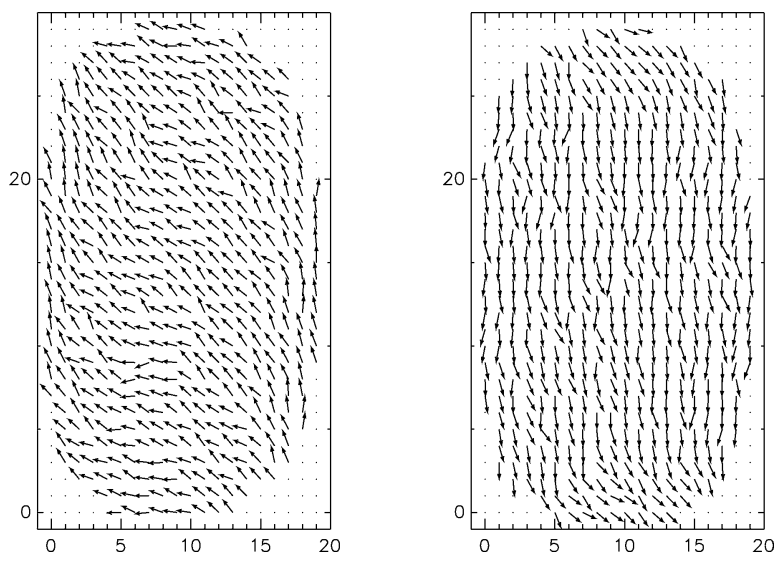

Fig. 2. A snap shot of the projection of the magnetization configuration onto the $x y$ plane when the external field is at -135 degrees. The left (right) panel is for the top (bottom) layer. Only every other spins in the $x$ and the $y$ directions are shown.

figuration of this run is taken as the initial configuration of a run with the magnetic field along the negative $y$ direction. After equilibrium is reached, we again take the final configuration of this run as the initial configuration of a run with the field oriented along the direction $\mathbf{v}=(1,-1) / \sqrt{2}$.

As the external field oriented at -135 degrees (along u) is increased, the edge domain in the top layer (left panel) expands while the bottom layer remains relatively unchanged. This is illustrated in Fig. 2. The asymmetric behaviour comes about because the external field is more aligned with the bottom layer. Note that for the top layer the magnetization near the left and the right edges remain relatively unchanged. These edgepinned domains are very similar to that observed in the single layer case [5]. For the bilayer situation, even though the top and bottom layer magnetizations are approximately opposite, the dipolar interaction from both layers reinforce each other to align the magnetization near the edge to be parallel to it. When the external field is changed to orient along the negative $y$ direction (Fig. 3), the top and bottom edge domains of the bottom layer expand while the magnetization of the top layer remains relatively unchanged. Finally, when the external field is at -45 degrees (Fig. 4), the top layer switched over if the field is strong enough. The switching scenario involves going through an intermediate state with edge-pinned domains, similar to the single layer case [5].

The temperature dependence of this switching field is shown in Fig. 5, together with that for a monolayer with identical parameters. For the bilayer system, the switching field is bigger. Furthermore, the temperature dependence is stronger! In the coherent rotation model, the activation energy is expected to be never small [2]. As a result, this model suggests that the temperature dependence of the switching field to be weaker. Our simulation result is opposite to the predictions based on the coherent rotation model. We emphasize that in the simulation, the parameters are the same at the different temperatures. Thus the temperature dependence of $H_{c}$ is not from possible additional temperature dependence of the physical parameters. The switching mechanism for the final step in Fig. 4 is illustrated in Fig. 6. It is initiated by the creation of a point-like defect in 

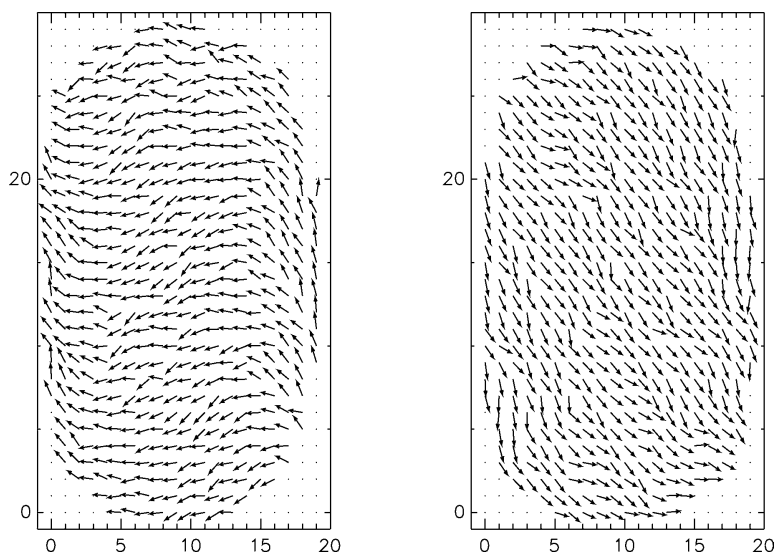

Fig. 3. A snap shot of the projection of the magnetization configuration onto the $x y$ plane when the external field is along the negative $y$ direction. The left (right) panel is for the top (bottom) layer. Only every other spins in the $x$ and the $y$ directions are shown.
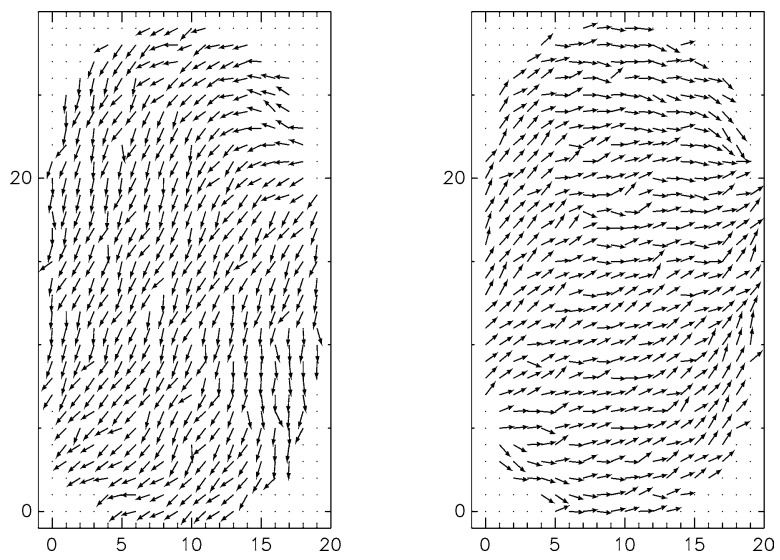

Fig. 4. A snap shot of the projection of the magnetization configuration onto the $x y$ plane when the external field is at -45 degrees. The left (right) panel is for the top (bottom) layer. Only every other spins in the $x$ and the $y$ directions are shown.

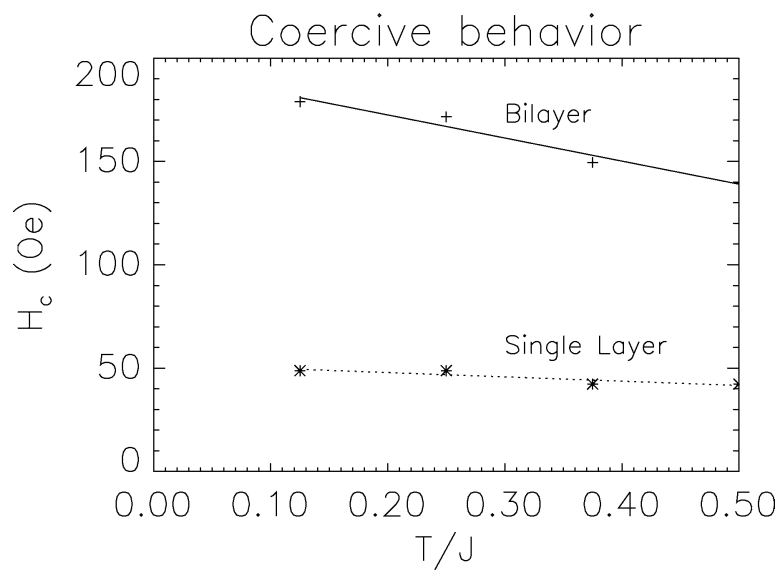

Fig. 5. The switching field for a bilayer and a single layer as a function of temperature $T$ normalized by the intralayer exchange $J$.

edge-pinned domain in the upper left corner. Physically, we expect the nucleation of this point defect to be thermally activated. Because of the antiferromagnetic coupling between the layers,
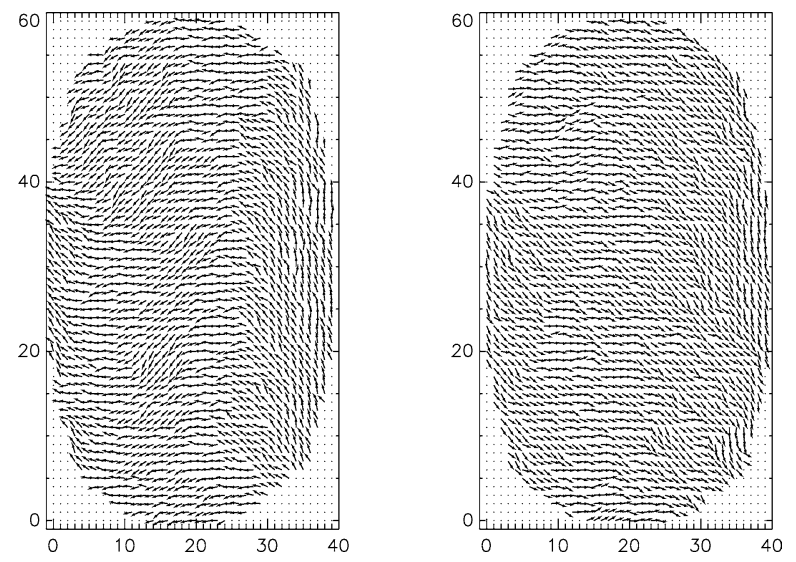

Fig. 6. A snap shot of the projection of the magnetization configuration onto the $x y$ plane during the switching when the external field is at -45 degrees. The left (right) panel is for the top (bottom) layer.

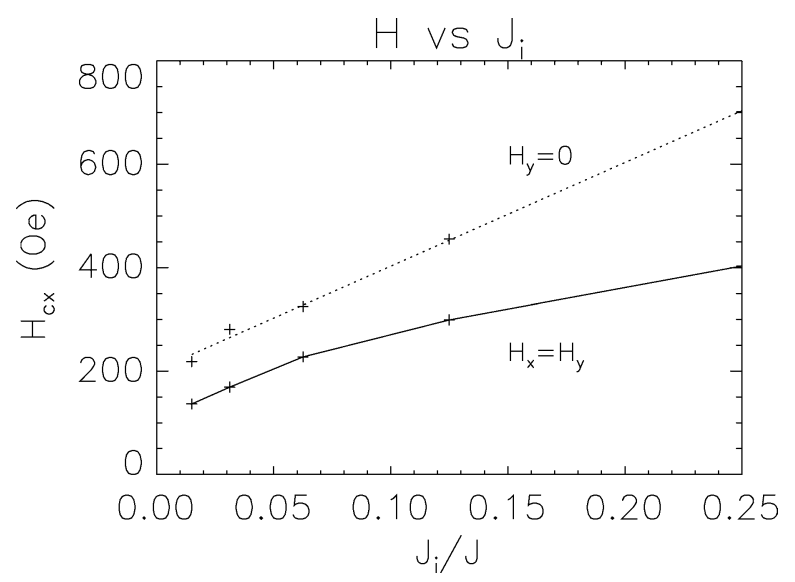

Fig. 7. The $u$ component of the switching fields for the case with $H_{u}=H_{v}$ and the case with $H_{v}=0$ as a function of the interlayer antiferromagnetic exchange constant $J_{i}$ normalized by the intraplane coupling.

the coupling of the individual magnetization to the external field is reduced. We write this coupling energy phenomenologically as $\gamma H M$. In the large $J_{i}$ limit, $\gamma \propto 1 / J_{i}$. In general we expect $\gamma \ll 1$. For a single layer the free energy of nucleation can be written as $F_{s}=\alpha\left[H_{c}(T=0)-H\right]^{\beta}$ for some constants $\alpha$, $\beta$. For a coupled layer, we expect the free energy to be given by $F=\alpha\left[\gamma\left(H_{c}(T=0)-H\right)\right]^{\beta}$. Following the usual argument [8], the nucleation probability $P$ in a time $\tau$ at temperature $T$ is given by $P=v \tau \exp (-F / k T)$ where $v$ is the spin flipping rate. The switching field is determined by the condition that $P=1$. We get $H_{c}(T)=H_{c}(T=0)-[k T \ln (\nu \tau) / \alpha]^{1 / \beta} / \gamma$. Because $\gamma \ll 1$, the temperature dependence is enhanced.

The dependence of the switching field on the interlayer exchange $J_{i}$ coupling is illustrated in Fig. 7. This switching field extrapolates to a finite value as the interlayer exchange extrapolates to zero. Part of the contribution of this finite value is due to the interlayer dipolar coupling. The interlayer dipolar coupling can in principle be reduced by increasing the interlayer separation. We have performed simulations with the $z$ separation increased by a factor of 10 and found that the switching field is reduced by only about 35 per cent. Thus a fairly large separation is required. 
Recall that the external fields are applied along the directions $\mathbf{u}=(-1,-1) / \sqrt{2}$ and $\mathbf{v}=(1,-1) / \sqrt{2}$. In Fig. 7 we show the switching boundaries for the $u$ component of the magnetic field for the case with $H_{u}=H_{v}$ as well as the case with $H_{v}=0$. Our calculations are carried out for perfect systems. For imperfect systems, there will be fluctuation of the switching field around these boundaries. The advantage of spin-flop switching is that if the interplane coupling is big enough these two boundaries are far apart so that fluctuations around one boundary do not overlap fluctuations around the other boundary. As a result, other bits will not be written accidentally. The separation between these boundaries decreases as the interplane coupling is decreased and the advantage of spin-flop switching is diminished. Thus the interplane coupling $J_{i}$ has to be large enough that the separation between the boundaries is larger than the sum of the fluctuation of these boundaries.

In Fig. 7, the interplane dipolar interaction is kept constant. As this coupling is decreased, we expect the two curves in Fig. 7 to come even closer.

Cowburn [3] and Korenivski and Worledge [3] recently reported results for circular samples that suggests the validity of the coherent rotation model. The calculation performed here is for the case when the aspect ratio is different from one and there is a finite shape anisotropy. For circular samples with the aspect ratio equal to one, the shape anisotropy is equal to zero and the effect described in this Letter may be absent.

\section{Acknowledgements}

This work was partially supported by the NSF. S.T.C. thanks the hospitality of the Physics Department and the Theory In- stitute of the National Taiwan University where this work was completed.

\section{References}

[1] B.N. Engel, et al., IEEE Trans. Magn. 41 (2005) 132; Savtchenko, et al., US pat No. 6545906 B1.

[2] D.C. Worledge, Appl. Phys. Lett. 84 (2004) 4559; D.C. Worledge, Appl. Phys. Lett. 84 (2004) 2847; F. Fujiwara, S.-Y. Wang, M. Sun, J. Appl. Phys. 97 (2005) 10 P507.

[3] R.P. Cowburn, J. Magn. Magn. Mater. 242 (2002) 505; V. Korenivski, D.C. Worledge, Appl. Phys. Lett. 86 (2005) 252506.

[4] N. Tezuka, N. Koike, K. Inomata, S. Sugimoto, J. Appl. Phys. 93 (2003) 7441.

[5] J. Shi, S. Tehrani, Appl. Phys. Lett. 77 (2000) 1692.

[6] S.T. Chui, Phys. Rev. B 55 (1997) 3688.

[7] S.T. Chui, Micromagnetism in small structures, in: NATO ASI Proceedings on Micromagnetism, Kluwer, Boston, 1996;

S.T. Chui, V. Ryzhov, Phys. Rev. Lett. 78 (1997) 2224;

S.T. Chui, V. Ryzhov, J. Magn. Magn. Mater. 177 (1998) 1303;

S.T. Chui, Appl. Phys. Lett. 68 (1996) 3641;

S.T. Chui, D.C. Tian, J. Appl. Phys. 78 (1995) 3965;

S.T. Chui, J. Appl. Phys. 79 (1995) 4951;

S.T. Chui, Phys. Rev. B 55 (1997) 3688;

S.T. Chui, J. Magn. Magn. Mater. 168 (1997) 9;

Y. Ma, S.T. Chui, J. Appl. Phys. 88 (2000) 1583.

[8] L.D. Landau, E.M. Liftshitz, Statistical Physics, Addison-Wesley, New York, 1969. 